\title{
Phonon-Assisted Tunneling of an Electron in a Strained Self-Assembled Quantum Dot Molecule
}

\author{
M. Pochwala and P. Machnikowski \\ Institute of Physics, Wrocław University of Technology \\ Wybrzeże Wyspiańskiego 27, 50-370 Wrocław, Poland
}

We calculate phonon-assisted relaxation rates in a self-assembled quantum dot molecule. The calculation is based on wave functions obtained within an envelope function approach, including strain effects. The single-phonon relaxation is shown to be efficient for separations between the dots around $10 \mathrm{~nm}$ when, in addition, the dots are brought close to resonance.

PACS numbers: 73.21.La, 73.63.Kv, 63.20.kd

\section{Introduction}

Carrier transfer and relaxation between quantum dots (QDs) are observed in many optical experiments [1-4]. So far, phonon assisted tunneling was theoretically studied for various electron configurations in lateral quantum dot molecules (QDMs) generated by electrostatic potentials [5-7]. For vertically stacked self-assembled structures, only a transfer process mediated by the Coulomb interaction was analyzed [8-11], which is relevant for relatively large separations between the dots. The problem with self-assembled structures is that the wave functions critically depend on the strain distribution. Since the tunneling rates are exponentially sensitive to the shape of the energy barrier between the two dots one has to include a realistic calculation of the electronic states.

Strain fields and the resulting electron states have been successfully calculated for single- and double QD structures [12-14]. However, for the carrierphonon interaction problem to be treatable, the electron wave functions must be known in a reasonably simple form and their computation should be possibly fast. As a trade-off between the requirements of simplicity and accuracy, we choose to use a kind of strain-dependent effective mass approach. Thus, we first calculate the strain distribution for a system of two dome-shaped, vertically stacked self-assembled InAs/GaAs QDs within the continuum elasticity approach in a cylindrical approximation. Next, we determine the "local band structure" at a given point from the 8-band $k p$ Hamiltonian, including the effects of the local strain, by perturbative eliminating the coupling to the hole bands. From this we extract the 
local electron effective mass and the local conduction band edge which is then used as the effective potential. The wave functions for the two lowest states in the QDM are calculated using a variational method based on the "adiabatic" separation of variables [15].

The electron wave functions obtained in this way are used for the calculation of the phonon-assisted tunneling rate between states localized in the two dots in the presence of an external electric field (which we include as a parameter that allows us to bring the dots to resonance). We show that the tunneling rate has a nontrivial dependence on the distance between the dots and on the energy difference between the confined states, which results from the interplay between the overlap of the wave functions and the splitting between the energy levels.

\section{Strain}

We consider an axially symmetric system composed of two dome-shaped QDs aligned along the growth axis of the semiconductor structure. The geometry of the system is shown in Fig. 1 . We set $R_{1}=R_{2}=14 \mathrm{~nm}, H_{1}=H_{2}=4 \mathrm{~nm}$, and $H_{\mathrm{WL}}=0.4 \mathrm{~nm}$.
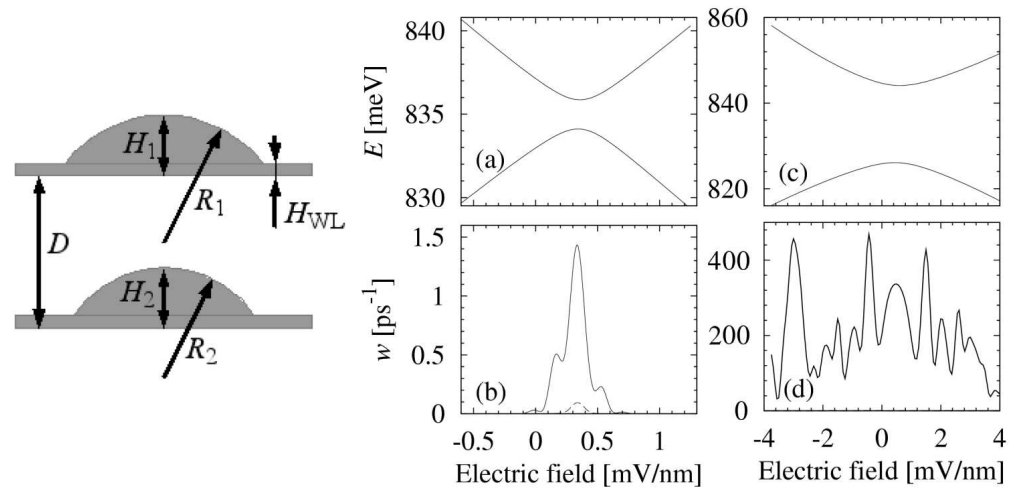

Fig. 1. (left) The geometry of the QDM structure. (right) The difference between the energy levels (a),(c) and the relaxation rates (b), (d) for structures with $D=120 \mathrm{~nm}$ (a),(b) and $D=80 \mathrm{~nm}$ (c),(d). In (b) and (d) the solid and dashed lines represent the contributions from the deformation potential coupling and from the piezoelectric coupling, respectively.

The strain distribution is calculated by minimizing the elastic energy of the inhomogeneous system of two InAs QDs in a GaAs crystal [13]:

$$
\begin{aligned}
E_{\mathrm{el}} & =\int \mathrm{d}^{3} r\left[\frac{1}{2} C_{11}\left(\epsilon_{x x}^{2}+\epsilon_{y y}^{2}+\epsilon_{z z}^{2}\right)+\frac{1}{2} C_{44}\left(\epsilon_{y z}^{2}+\epsilon_{z x}^{2}+\epsilon_{x y}^{2}\right)\right. \\
& \left.+C_{12}\left(\epsilon_{y y} \epsilon_{z z}+\epsilon_{y y} \epsilon_{x x}+\epsilon_{x x} \epsilon_{z z}\right)-\alpha\left(\epsilon_{x x}+\epsilon_{y y}+\epsilon_{z z}\right)\right] .
\end{aligned}
$$

Here $\epsilon_{i j}$ are the Cartesian components of the strain tensor, $C_{i j}$ are position- 
-dependent elastic constants, $\alpha=\left(C_{11}+2 C_{12}\right)\left(a_{\mathrm{I}} / a_{\mathrm{G}}-1\right)$ in a $\mathrm{QD}$ and $\alpha=0$ in GaAs, where $a_{\mathrm{I}}$ and $a_{\mathrm{G}}$ are the lattice constants of InAs and GaAs, respectively. The last term in Eq. (1) accounts for the mismatch of lattice constants, shifting the equilibrium of the InAs crystal lattice to the state appropriately stretched with respect to the ideal InAs crystal. Since the strain is calculated with respect to the GaAs lattice and GaAs crystal coordinates are used, the results for the InAs dots must be rescaled to yield physical strain, according to $\epsilon_{i j}=\left(a_{\mathrm{G}} / a_{\mathrm{I}}\right) \epsilon_{i j}-\delta_{i j}\left(1-a_{\mathrm{G}} / a_{\mathrm{I}}\right)[13]$. The values of the material parameters are taken from Ref. [12].

For an axially symmetric structure, it is convenient to perform the computation in cylindrical coordinates. Therefore we denote the components of the displacement in the local reference frame as $u_{r}, u_{\phi}, u_{z}$ and define the corresponding components of the strain tensor,

$$
\begin{aligned}
& \epsilon_{r r}=\frac{\partial u_{r}}{\partial r}, \quad \epsilon_{\phi \phi}=\frac{1}{r}\left(\frac{\partial u_{\phi}}{\partial \phi}+u_{r}\right), \quad \epsilon_{r \phi}=u_{\phi r}=\frac{1}{2 r}\left(\frac{\partial u_{r}}{\partial \phi}-u_{\phi}\right), \\
& \epsilon_{r z}=\epsilon_{z r}=\frac{1}{2}\left(\frac{\partial u_{r}}{\partial z}+\frac{\partial u_{z}}{\partial r}\right), \quad \epsilon_{\phi z}=\epsilon_{z \phi}=\frac{1}{2}\left(\frac{\partial u_{\phi}}{\partial z}+\frac{1}{r} \frac{\partial u_{z}}{\partial \phi}\right) .
\end{aligned}
$$

We will look for the minimum of $E_{\mathrm{el}}$ in the class of axially symmetric displacement fields, that is, $u_{\phi}=0$ and $\partial u_{r} / \partial \phi=\partial u_{z} / \partial \phi=0$. This is an approximation, since the crystal actually does not have the full rotational symmetry. However, a standard calculation in the Cartesian coordinates shows that both the angular dependence of the radial and axial displacements $u_{r}, u_{z}$ and the magnitude of the twisting displacement $u_{\phi}$ are negligibly small. With this approximation, the integration over $\phi$ in Eq. (1) can be performed analytically and one gets

$$
\begin{aligned}
E_{\mathrm{el}} & =\pi \int \mathrm{d} r r \int \mathrm{d} z\left[C_{11} \epsilon_{z z}^{2}+D\left(\epsilon_{r r}^{2}+\epsilon_{\phi \phi}^{2}\right)+C_{44} \epsilon_{r z}^{2}+F \epsilon_{r r} \epsilon_{\phi \phi}\right. \\
& \left.+2 C_{12} \epsilon_{z z}\left(\epsilon_{r r}+\epsilon_{\phi \phi}\right)-2 \alpha\left(\epsilon_{r r}+\epsilon_{\phi \phi}+\epsilon_{z z}\right)\right],
\end{aligned}
$$

where $D=3 C_{11} / 4+C_{12} / 4+C_{44} / 8$ and $F=C_{11} / 4+3 C_{12} / 4-C_{44} / 8$.

The displacement field minimizing $E_{\mathrm{el}}$ is found by the conjugate gradient method on a square grid of 500 points along $z$ and 333 points along $r$, representing a cylinder with the height of $30 \mathrm{~nm}$ and the radius of $200 \mathrm{~nm}$. A combination of discretizations with forward and backward representations of derivatives is used to avoid discretization-induced oscillations [13].

\section{Electron states}

In order to find approximate electron wave functions in the double-dot structure, we use the envelope function approach [16] combined with a variational method which is an extension of the method of "adiabatic" separation of variables [15]. The first step is the determination of the local conduction band edge and the electron effective mass tensor at each point of the structure. The assumption 
here is that system parameters do not change too strongly over distances of the order of the lattice constant. One considers a crystal with uniform composition and strain values such as those at the considered point of the structure and uses its conduction band parameters as local values in the inhomogeneous heterostructure.

The band structure in a strained system is determined from the 8-band $\boldsymbol{k} \cdot \boldsymbol{p}$ (Kane) Hamiltonian with strain-induced terms (Bir-Pikus Hamiltonian) using the Löwdin elimination [17]. The part of the Hamiltonian coupling conduction and valence band states is [18]:

$$
\begin{aligned}
H_{\mathrm{c}-\mathrm{v}} & =A(|\mathrm{e} \uparrow\rangle\langle\mathrm{e} \uparrow|+| \mathrm{e} \downarrow\rangle\langle\mathrm{e} \downarrow|)+\left\{| \mathrm { e } \uparrow \rangle \left[-\sqrt{3} \mathrm{~V}^{\dagger}\langle\mathrm{hh} \uparrow|\right.\right. \\
& -U(\sqrt{2}\langle\mathrm{lh} \uparrow|-\langle\mathrm{so} \uparrow|)-V(\langle\mathrm{lh} \downarrow|-\sqrt{2}\langle\mathrm{so} \downarrow|)] \\
+ & |\mathrm{e} \downarrow\rangle[-\sqrt{3} \mathrm{~V}\langle\mathrm{hh} \downarrow|-\mathrm{U}(\sqrt{2}\langle\mathrm{lh} \downarrow|+\langle\mathrm{so} \downarrow|) \\
+ & \left.\left.V^{\dagger}(\langle\mathrm{lh} \uparrow|+\sqrt{2}\langle\text { so } \uparrow|)\right]+ \text { h.c. }\right\},
\end{aligned}
$$

where "lh", "hh", and "so" denote the heavy-hole, light-hole and split-off subbands, $\uparrow$ and $\downarrow$ represents the spin orientation in a given subband, $A=E_{\mathrm{c}}+$ $(\hbar k)^{2} /\left(2 m_{0}\right)+a_{\mathrm{c}} h, E_{\mathrm{c}}$ is the conduction band edge, $h=\operatorname{Tr} \epsilon$ is the hydrostatic strain, $U=P_{0}\left(k_{z}+\sum_{j} \epsilon_{j z} k_{j}\right) / \sqrt{3}$, and $V=P_{0}\left[k_{x}-\mathrm{i} k_{y}-\sum_{j}\left(\epsilon_{x j}-\epsilon_{y j}\right) k_{j}\right] / \sqrt{6}$. The diagonal terms for the valence band states are $E_{\mathrm{hh}}=E_{\mathrm{v}}-p-q, E_{\mathrm{lh}}=E_{\mathrm{v}}-p+q$, and $E_{\mathrm{so}}=E_{\mathrm{v}}-\Delta-p$, where $E_{\mathrm{v}}$ is the valence band edge of an unstrained crystal, $\Delta$ is the spin-orbit split-off parameter, $p=a_{\mathrm{v}} h, q=b\left[\epsilon_{z z}-(1 / 2)\left(\epsilon_{r r}+\epsilon_{\phi \phi}\right)\right]$, and the band structure parameters $E_{\mathrm{v}}, E_{\mathrm{c}}, E_{\mathrm{so}}, P_{0}, \Delta$ and deformation potentials $b, d$ are taken from Ref. [12]. Neglecting the strain-related terms in $U$ and $V$, which are much smaller than the purely kinetic ones, we get the conduction band energy up to the 2nd order in $\boldsymbol{k}, E(\boldsymbol{k})=E_{\mathrm{c}}+a_{\mathrm{c}} h+\hbar^{2} k_{\perp}^{2} /\left(2 m_{\perp}\right)+\hbar^{2} k_{z}^{2} /\left(2 m_{z}\right)$, where the in-plane and $z$ components of the effective mass tensor are

$$
\begin{aligned}
& m_{\perp}^{-1}=m_{0}^{-1}\left(1+\frac{E_{\mathrm{P}}}{2 \Delta E_{\mathrm{hh}}}+\frac{E_{\mathrm{P}}}{6 \Delta E_{\mathrm{lh}}}+\frac{E_{\mathrm{P}}}{3 \Delta E_{\mathrm{so}}}\right), \\
& m_{z}^{-1}=m_{0}^{-1}\left(1+\frac{2 E_{\mathrm{P}}}{3 \Delta E_{\mathrm{lh}}}+\frac{E_{\mathrm{P}}}{3 \Delta E_{\mathrm{so}}}\right),
\end{aligned}
$$

where $E_{\mathrm{P}}=2 m_{0} P_{0}^{2} / \hbar^{2}, \Delta E_{i}=E_{\mathrm{c}}+a_{\mathrm{c}} h-E_{i}, i=\mathrm{hh}, \mathrm{lh}$, so.

The envelope function of an electron is found from the Schrödinger equation with the Hamiltonian

$$
\begin{aligned}
H= & -\frac{\partial}{\partial x} \frac{\hbar^{2}}{2 m_{\perp}(r, z)} \frac{\partial}{\partial x}-\frac{\partial}{\partial y} \frac{\hbar^{2}}{2 m_{\perp}(r, z)} \frac{\partial}{\partial y}-\frac{\partial}{\partial z} \frac{\hbar^{2}}{2 m_{z}(r, z)} \frac{\partial}{\partial z} \\
& +E_{\mathrm{c}}(r, z)-e \mathcal{E} z,
\end{aligned}
$$

where we included an external electric field $\mathcal{E}$. Following the concept of "adiabatic" separation of variables, we first numerically solve the one-dimensional equation 
along the strongest confinement direction at each $r$,

$$
\left[-\frac{\partial}{\partial z} \frac{\hbar^{2}}{2 m_{z}(r, z)} \frac{\partial}{\partial z}+E_{\mathrm{c}}(r, z)-e \mathcal{E} z\right] \chi(r, z)=E(r) \chi(r, z) .
$$

Of interest are the lowest two solutions to this equation, representing the lowest subband of confined states in the double well system. Next, we apply the Ritz variational method [19], looking for the stationary points of the functional $F[\psi]=\langle\psi|H| \psi\rangle$ in the class of ansatz functions $\psi(r, z)=\sum_{i} \chi_{i}(r, z) \varphi_{i}(r)$. We first discretize the functional on the same lattice that was used in the computation of the strain and then derive an eigenvalue problem for the two-component wave function $\varphi_{i}(r), r=1,2$ (already in the discretized form) via stationarity requirement with respect to the values at the discrete points. This procedure allows for mixing of the two manifolds of states related to the two functions $\chi_{1}(r, z)$ and $\chi_{2}(r, z)$, which is essential when the two QDs are of similar size or when the thinner dot has a larger in-plane size, so that a crossing of the one-dimensional solutions appears at a certain $r$.

\section{Phonon-assisted relaxation}

The rate for the phonon-assisted relaxation is found from the Fermi golden rule formula $[20]$ :

$$
w=\frac{2 \pi}{\hbar} \sum_{\boldsymbol{k}}\left|F_{s}(\boldsymbol{k})\right|^{2}\left[n_{\mathrm{B}}\left(\omega_{\boldsymbol{k}}\right)+1\right] \delta(h \omega k-\Delta E),
$$

where $\Delta E$ is the energy difference between the two electron states, $\boldsymbol{k}$ and $s$ are a phonon wave vector and polarization $(s=1$ for longitudinal, $s=\mathrm{t} 1$, $\mathrm{t} 2$ for the two transverse ones), $\omega_{\boldsymbol{k}, s}$ is the corresponding frequency, $n_{\mathrm{B}}(\omega)=$ $\left[\exp \left(\hbar \omega /\left(k_{\mathrm{B}} T\right)\right)-1\right]^{-1}$ is the Bose distribution at the temperature $T$, and $F_{s}(\boldsymbol{k})$ are the carrier-phonon coupling constants. The latter have the form $F_{s}(\boldsymbol{k})=$ $\left(v_{\boldsymbol{k}, s}^{(\mathrm{DP})}+v_{\boldsymbol{k}, s}^{(\mathrm{P})}\right) \mathcal{F}(\boldsymbol{k})$, where

$$
\mathcal{F}(\boldsymbol{k})=\int \mathrm{d}^{3} r \psi_{1}^{*}(\boldsymbol{r}) \mathrm{e}^{\mathrm{i} \boldsymbol{k} \cdot \boldsymbol{r}} \psi_{2}(\boldsymbol{r})
$$

and the coefficients describing the electron-phonon coupling via deformation potential and via Coulomb interaction with the phonon-induced polarization field are, respectively,

$$
v_{\boldsymbol{k}, l}^{(\mathrm{DP})}=\sqrt{\frac{\hbar k}{2 \rho V c_{1}}} \sigma, \quad v_{\boldsymbol{k}, s}^{(\mathrm{P})}=-\mathrm{i} \sqrt{\frac{\hbar}{2 \rho V c_{s} k}} \frac{d e}{\epsilon_{0} \epsilon_{\mathrm{r}}} M_{s}(\hat{\boldsymbol{k}}),
$$

where $c_{s}$ is the speed of sound for the phonon branch $s\left(c_{1}=5150 \mathrm{~m} / \mathrm{s}\right.$, $\left.c_{\mathrm{t}}=2800 \mathrm{~m} / \mathrm{s}\right), \rho=5300 \mathrm{~kg} / \mathrm{m}^{3}$ is the crystal density, $V$ is the normalization volume for the phonon modes, $\sigma=-9 \mathrm{eV}$ is the deformation potential constant, $d=0.16 \mathrm{C} / \mathrm{m}^{2}$ is the piezoelectric coupling constant, $e$ is the elementary charge, 
and $M_{s}(\boldsymbol{k})=2\left[k_{x} k_{y}\left(\hat{e}_{\boldsymbol{k}, s}\right)_{z}, k_{y} k_{z}\left(\hat{e}_{\boldsymbol{k}, s}\right)_{x}, k_{z} k_{x}\left(\hat{e}_{\boldsymbol{k}, s}\right)_{y}\right]$ for a zinc-blende crystal. Deformation potential is zero for transverse modes.

We apply the theory developed in the previous section to the InAs/GaAs QDM. In Figs. 1a,c we show the two lowest energy levels as a function of the electric field for two different values of the distance between the dots (left: $120 \mathrm{~nm}$, right: $80 \mathrm{~nm}$ ). In each case, a clear anticrossing of the levels is visible. The width of the anticrossing grows very fast when the distance between the dots decreases.

The electron relaxation rate for the two QDM structures is plotted in Figs. 1b,d. For $D=120 \mathrm{~nm}$, the energy difference at the resonance corresponds to the range of high phonon spectral density. This results in fast relaxation on ps time scales. Exactly at the resonance and in a certain range of electric fields around the resonance, such that the energy difference is comparable with the anticrossing width, the wave functions have predominantly symmetric-antisymmetric character. Therefore, the phonon-assisted process is a relaxation between two delocalized states with no (or weak) transfer of the electron occupation between the dots. Further away from the resonance, the eigenstates correspond to an electron localized in one of the dots. Although the relaxation rate decreases considerably as soon as the energies are detuned from the resonance, the relaxation remains relatively fast (tens of picoseconds) also outside the resonance sector. In this case the process has the character of phonon-assisted tunneling, consisting in an inelastic transfer from the higher energy dot to the lower energy one. Comparison between the contribution originating from the two coupling mechanisms shows that the deformation potential coupling dominates over the piezoelectric one.

When the dots are placed at a smaller distance, the tunnel coupling considerably increases the energy splitting (see Fig. 1c). Since the wave vectors (and hence also energies) of phonons effectively coupled to a confined charge are limited [20], the increased energy splitting suppresses the relaxation processes by many orders of magnitude. The contribution from the piezoelectric coupling is now three orders of magnitude smaller than that from the deformation potential coupling.

\section{Conclusion}

We have calculated phonon-assisted relaxation rates in a self-assembled quantum dot molecule, including strain effects. Relaxation rates are very high (picosecond range) for delocalized states near resonance in a narrow range of dot separations around $10 \mathrm{~nm}$. At such separations, relaxation remains relatively fast (10-100 ps) also out of resonance, where the states are localized in different dots (phonon-assisted tunneling process). Under such conditions, relaxation is dominated by deformation potential coupling with longitudinal acoustic (LA) phonons. For smaller separations (below $10 \mathrm{~nm}$ ), large tunnel coupling shifts the states away from each other and the relaxation becomes inefficient due to limited energies of effectively coupled acoustic phonons. In both cases, relaxation is dominated by deformation potential coupling to LA phonons. 


\section{References}

[1] S. Rodt, V. Turck, R. Heitz, F. Guffarth, R. Engelhardt, U.W. Pohl, M. Straßburg, M. Dworzak, A. Hoffmann, D. Bimberg, Phys. Rev. B 67, 235327 (2003).

[2] B.D. Gerardot, S. Strauf, M.J.A. de Dood, A.M. Bychkov, A. Badolato, K. Hennessy, E.L. Hu, D. Bouwmeester, P.M. Petroff, Phys. Rev. Lett. 95, 137403 (2005).

[3] G. Ortner, R. Oulton, H. Kurtze, M. Schwab, D.R. Yakovlev, M. Bayer, S. Fafard, Z. Wasilewski, P. Hawrylak, Phys. Rev. B 72, 165353 (2005).

[4] T. Nakaoka, E.C. Clark, H.J. Krenner, M. Sabathil, M. Bichler, Y. Arakawa, G. Abstreiter, J.J. Finley, Phys. Rev. B 74, 121305(R) (2006).

[5] V.N. Stavrou, X. Hu, Phys. Rev. B 72, 75362 (2005).

[6] S. Vorojtsov, E.R. Mucciolo, H.U. Baranger, Phys. Rev. B 71, 205322 (2005).

[7] A. Grodecka, P. Machnikowski, J. Förstner, arXiv:0803.1734v1 [cond-mat.meshall], unpublished results.

[8] A.O. Govorov, Phys. Rev. B 68, 075315 (2003).

[9] M. Richter, K.J. Ahn, A. Knorr, A. Schliwa, D. Bimberg, M.E.-A. Madjet, T. Renger, Phys. Status Solidi B 243, 2302 (2006).

[10] E. Rozbicki, P. Machnikowski, Acta Phys. Pol. A 112, 197 (2007), arXiv:0706.0862 [cond-mat.mes-hall].

[11] E. Rozbicki, P. Machnikowski, Phys. Rev. Lett. 100, 027401 (2008).

[12] C. Pryor, Phys. Rev. B 57, 7190 (1998).

[13] C. Pryor, J. Kim, L.W. Wang, A.J. Williamson, A. Zunger, J. Appl. Phys. 83, 2548 (1998).

[14] M. Korkusinski, P. Hawrylak, Phys. Rev. B 63, 195311 (2001).

[15] A. Wójs, P. Hawrylak, S. Fafard, L. Jacak, Phys. Rev. B 54, 5604 (1996).

[16] G. Bastard, Wave Mechanics Applied to Semiconductor Heterostructures, Editions de Physique, Les Ulis 1988.

[17] P.O. Löwdin, J. Chem. Phys. 19, 1396 (1951).

[18] T.B. Bahder, Phys. Rev. B 41, 11992 (1990).

[19] A. Messiah, Quantum Mechanics, North-Holland, Amsterdam 1966.

[20] L. Jacak, P. Machnikowski, J. Krasnyj, P. Zoller, Eur. Phys. J. D 22, 319 (2003). 